\title{
Efecto de mezclas bacterianas con potencial probiótico en el crecimiento, supervivencia y respuesta antioxidante de la almeja catarina Argopecten ventricosus
}

\author{
Effect of bacterial mixtures with probiotic potential on catarina scallop Argopecten ventricosus growth, \\ survival and antioxidant response
}

\author{
Fernando Abasolo-Pacheco ${ }^{1,2 *}$, Pedro E. Saucedo', José M. Mazón-Suástegui', Ángel I. Campa-Córdova', Yarelys \\ Ferrer-Sánchez ${ }^{2}$, Yuniel Méndez-Martínez ${ }^{2}$, Nora Fernanda Chiluisa Triana² \\ Centro de Investigaciones Biológicas del Noroeste, La Paz, B.C.S. 23096, México \\ 2 Universidad Técnica Estatal de Quevedo (UTEQ), Quevedo, Los Ríos EC.120501, Ecuador.
}

\section{RESUMEN}

El uso de bacterias probióticas como agentes de control biológico ha sido efectivo, sin embargo, los trabajos se han centrado en la aplicación de manera individual, sin tomar en cuenta que las mezclas podrían potenciar los efectos. En este trabajo se evaluaron mezclas de bacterias con potencial probiótico sobre larvas y juveniles de Argopecten ventricosus. Los organismos se cultivaron en condiciones de laboratorio y fueron tratados con seis mezclas de Bacillus y Lactobacillus en dos diferentes concentraciones. Se incluyó un grupo de organismos tratados con ampicilina y otro sin tratamiento como control. El tratamiento con la mezcla de Lactobacillus y Bacillus (MIX-LBC2) presentaron una respuesta significativa en las variables supervivencia, crecimiento y fijación de larvas. En juveniles el mayor crecimiento se observó con la mezcla de Lactobacillus (MIX-LC2) y la mezcla con Bacillus y Lactobacillus (MIX-LBC1). Durante el reto experimental con V. alginolyticus, la mezcla de Bacillus (MIX-BC2) aumentó significativamente la supervivencia de juveniles, lo que concuerda con la actividad de la enzima superóxido dismutasa. Los resultados indican que el mecanismo de acción de las mezclas con bacterias probióticas es específico de la etapa y la cepa, mejorando la supervivencia, el crecimiento y mayor resistencia contra el patógeno.

Palabras clave: acuicultura, bivalvos, cultivo, salud, mezcla de cepas probióticas

\section{ABSTRACT}

The use of probiotic bacteria as biological control agents has been effective; however, most work has focused on the individuall application, without taking into account that the mixtures could enhance the effects. In this work, we evaluated the effect of probiotics mixtures on Argopecten ventricosus larvae and juveniles. Organisms cultured under laboratory conditions were treated with six Bacillus and Lactobacillus mix at two different concentrations. A group of ampicillin-treated organisms and a group without treatment as control were included. Significantly higher survival, size growth and larval fixation of early veliger larvae occurred with the mix of Lactobacillus and Bacillus (MIX-LBC2), while higher juveniles growth was observed with the Lactobacillus

\section{Volumen XXI, Número 3}

mixture (MIX-LC2) and the mix with Bacillus and Lactobacillus (MIX-LBC1). During challenge with V. alginolyticus, the Bacillus mix (MIX-BC2) significantly increased the juveniles survival, consistent with maximum activity of superoxide dismutase enzyme. Results indicate that the probiotics mix mechanism of action is specific to the stage and strain, generating different responses by the host, including improved survival and growth and higher resistance against the pathogen.

Keywords: aquaculture, bivalves, hatchery-rearing, health, mix probiotic strains.

\section{INTRODUCCIÓN}

El cultivo de bivalvos marinos, incluido el de la almeja Catarina Argopecten ventricosus (Sowerby II 1842), representa un recurso altamente valorado para el consumo humano en el noroeste de México (Maeda-Martínez, 2008); sin embargo, el cultivo de este género se ve afectado por la incidencia de patógenos marinos que causan altas mortalidades en larvas y juveniles (Caceres y Vazquez, 2008; Romalde, 2016). Uno de los principales patógenos de esta especie está representado por bacterias del genero Vibrio y particularmente Vibrio alginolyticus (Luna-González et al., 2002; Cordero et al., 2014). El tratamiento de organismos cultivados con terapias más naturales y ecológicamente amigables, como los probióticos, continúa siendo viable para el control o erradicación de los patógenos (Balcázar et al., 2008). Con lo anterior se busca eliminar o disminuir el uso de antibióticos los cuales han provocado resistencia bacteriana (Alós, 2015) y la contaminación ambiental por el desecho al medio de las aguas tratadas con estas sustancias (Lawrence y Jeyakumar, 2013).

Entre los efectos benéficos de bacterias tenemos la síntesis de compuestos antimicrobianos y enzimas digestivas que mejoran la asimilación de nutrientes del hospedero y también mejoran la respuesta del sistema inmune y la capacidad de tolerancia bajo condiciones de estrés (Verschuere et al., 2000). El conocimiento científico actual sobre el uso de probióticos es escaso en moluscos bivalvos, pero el creciente interés y los avances recientes en este campo demuestran su importancia (Kesarcodi-Watson et al., 2008; Sánchez-Ortiz et al., 2015; Abasolo-Pacheco et al., 2016). La mayoría de investigaciones en el campo de la acuicultura han

*Autor para correspondencia: Dr. Fernando Abasolo Pacheco Correo electrónico: fabasolo@uteq.edu.ec 
involucrado la aplicación de bacterias benéficas de manera individual, sin embargo, las mezclas de estas, pueden funcionar mejor que individualmente (Gatesoupe, 2002). En este sentido, la aplicación de probióticos como preparaciones en mezclas proporciona beneficios mejorados en los bivalvos (Kesarcodi-Watson et al., 2012; Abasolo-Pacheco et al., 2016), particularmente las bacterias del género Bacillus spp. y Lactobacillus spp. (Nimrat et al., 2012; Yeh et al., 2014). Así mismo, la relación dosis-efecto de un probiótico se debe determinar cuidadosamente con la finalidad de evitar una sobredosis, lo cual puede resultar en una disminución en su efecto y un incremento en los costos, o contrariamente, usar dosis muy bajas que podrían reducir la eficacia del probiótico (Vine et al., 2006).

Por lo anterior, el objetivo del presente estudio fue determinar el efecto de mezclas de cepas probióticas de Bacillus spp. y Lactobacillus spp., en dos diferentes concentraciones $\left(1 \times 10^{5}\right.$ UFC $\mathrm{mL}^{-1}$ y $\left.1 \times 10^{6} \mathrm{UFC} \mathrm{mL}^{-1}\right)$ sobre el crecimiento de $A$. ventricosus y la supervivencia y respuesta antioxidante durante un reto experimental con la cepa patógena Vibrio alginolyticus CAIM57.

\section{MATERIALES Y MÉTODOS}

\section{Origen de larvas y juveniles de Argopecten ventricosus}

Las larvas se obtuvieron usando protocolos estándar para cultivo de esta especie (Mazón-Suástegui, 2005). El cultivo de larvas se llevó a cabo en tanques de fibra de vidrio de $1500 \mathrm{~L}$, por triplicado, llenados con agua de mar filtrada a $1 \mu \mathrm{m}$ y esterilizada con UV a $23 \pm 1^{\circ} \mathrm{C}$ y una salinidad de $37 \pm 0.5$ ups (unidades prácticas de salinidad). Las larvas se sembraron en una relación de 8 larvas $\mathrm{mL}^{-1}$ y se alimentaron con Isochrysis galbana (v. Aff galbana, código UTEX LB 2307) del día 1 al 8 y una mezcla 1: 1 de l. galbana y Chaetoceros calcitrans (IFREMER) desde el día 8 en adelante. El establecimiento de larvas de pediveliger se produjo en colectores artificiales hechos de bolsas de fibra de plástico y sumergidas dentro de los tanques de cultivo. Después de dos semanas, todos los juveniles que se asentaron en el fondo, colectores y paredes de los tanques de cultivo se separaron y se transfirieron a unidades de Pre-engorda de semillas de moluscos, para su uso posterior en los bioensayos con juveniles.

\section{Obtención de bacterias probióticas}

Las bacterias previamente seleccionadas (AbasoloPacheco et al., 2016) fueron reactivadas en caldo Man Rogosa Sharp (MRS; \#288110, Bioxon BD Difco, Franklin Lakes, NJ) para el caso de los Lactobacillus spp. y Trypticase Soy Broth (TSB; \#257107, Bioxon BD Difco) para el caso de Bacillus spp. Ambas especies se incubaron a $35^{\circ} \mathrm{C}$ por 24 horas. Posteriormente fueron centrifugadas $\left(4696 \times g\right.$ por $10 \mathrm{~min}$ a $4^{\circ}$ C), y resuspendidas en agua de mar estéril y ajustadas a una densidad óptica de $1.0(540 \mathrm{~nm})$ para obtener una densidad de $\sim 1 \times 10^{9} \mathrm{CFU} \mathrm{mL}{ }^{-1}$, de acuerdo a la escala de McFarland. Finalmente, esta densidad fue ajustada a las diferentes concentraciones que se utilizaron en los bioensayos con larvas $y$ juveniles $\left(\mathrm{C} 1: 1 \times 10^{5} \mathrm{CFU} \mathrm{mL}^{-1}\right.$ y $\left.\mathrm{C} 2: 1 \times 10^{6} \mathrm{CFU} \mathrm{mL}^{-1}\right)$.

\section{Bioensayo I. Supervivencia, crecimiento y fijación larva- ria de Argopecten ventricosus}

Para este bioensayo se colocaron larvas velíger (81.5 $\pm 1 \mu \mathrm{m}$ de altura de la concha) en unidades experimentales plásticas de $4 \mathrm{~L}$ con agua de mar filtrada $(1 \mu \mathrm{m})$ y esterilizada con UV. La temperatura del bioensayo fue de $23^{\circ} \mathrm{C} \pm 1^{\circ} \mathrm{C}$ y 37 ups de salinidad. Cada uno de las unidades contenía $16 \times 10^{3}$ larvas a una densidad de 8 larvas $\mathrm{mL}^{-1}$ y fueron tratadas con diferentes mezclas de bacterias con potencial probiótico, en dos concentraciones diferentes, $\mathrm{C} 1: 1 \times 10^{5} \mathrm{UFC} \mathrm{mL}{ }^{-1}$ y C2: 1

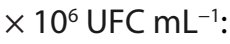

(1) MIX-LC1 (L. plantarum C3 + L. graminis RL5);

(2) MIX-BC1 (Bacillus cereus PB1-1 + B. flexus PB1-5 + B. firmus PB1-6);

(3) MIX-LBC1 (L. plantarum C3 + L. graminis RL5 + Bacillus cereus PB1-1 + B. flexus PB1-5 + B. firmus PB1-6);

(4) MIX-LC2;

(5) MIX-BC2;

(6) MIX-LBC2;

(7) ampicilina 10 ppm (AMP);

(0) CTRL (grupo control, sin ampicilina y sin bacterias benéficas).

Todos los tratamientos se realizaron por triplicado. Todos los grupos de larvas recibieron las microalgas primero y posteriormente las bacterias. Los tratamientos se suministraron directamente en el agua cada $48 \mathrm{~h}$. Este bioensayo tuvo una duración de siete días.

Al inicio del bioensayo, se fotografiaron con ayuda de una cámara digital y un microscopio compuesto marca Olympus BX-41 (Olympus Corporation Inc. Tokio, Japón) 30 larvas velíger de cada réplica de los tratamientos y las imágenes fueron procesadas con el software Image Pro Plus 6.0 (Media Cybernetics, Bethesda, MD, E.U.A.) para determinar la altura de las larvas $(0.1 \mu \mathrm{m})$. Para las mediciones posteriores se tomaron muestras al azar de 30 larvas por tratamiento en los días 3,5 y 7 . Con estos datos se determinó la supervivencia (\%), crecimiento absoluto $(\mu \mathrm{m})$ y tasa de crecimiento $(\mu \mathrm{m}$ $\mathrm{d}^{-1}$ ). Para determinar la supervivencia se utilizó una cámara de conteo Sedgewick Rafter, para estimar el total de larvas vivas. El crecimiento se determinó con la siguiente ecuación:

$$
\text { Tasa de crecimiento }(t c)=\frac{\text { Tfinal }- \text { Tinicial }}{t}
$$

Donde: $T=$ Talla inicial y final del tiempo de experimento $\mathrm{t}=$ tiempo en días

Después de la fijación, se realizaron los conteos correspondientes por cada réplica, para determinar el rendimiento total por tratamiento, los datos se expresaron como porcentaje (\%) de juveniles reclutados.

\section{Bioensayo II. Supervivencia y crecimiento de juveniles de Argopecten ventricosus}

El diseño experimental fue similar al descrito en la sección anterior, pero ahora se incluyeron 3,600 juveniles de 
A. ventricosus (48 días de edad; $4.14 \pm 0.06 \mathrm{~mm}$ de altura de la concha; $13.33 \pm 0.03 \mathrm{mg}$ de peso húmedo; $6.6 \pm 0.03 \mathrm{mg}$ de peso seco). Los juveniles fueron divididos en ocho grupos experimentales de 120 almejas cada uno, se alimentaron diariamente con una mezcla 1:1 de las microalgas I. Galbana y Chaetoceros calcitrans a una concentración de $150 \times 10^{3}$ células $\mathrm{mL}^{-1}$, y con los tratamientos de bacterias correspondientes (por triplicado). El bioensayo tuvo una duración de 21 días.

Al inicio del bioensayo, se tomaron 30 muestras al azar de cada una de las réplicas de los grupos experimentales, se tomaron fotos y se procesaron con el software Image Pro Plus 6.0 software para determinar la altura inicial de la concha $( \pm 0.1 \mathrm{~mm})$. Los juveniles también fueron pesados en una balanza analítica ( $0.1 \mathrm{~g}$ de precisión) marca Ohaus ${ }^{\circ}$ (con concha) para estimar su peso húmedo y peso seco inicial $( \pm 0.1 \mathrm{~g})$. Para las posteriores mediciones se tomaron muestras al azar de 30 juveniles por tratamiento en los días 7,14 y 21. Con estos datos se determinó la supervivencia (\%), crecimiento absoluto $(\mathrm{mm}, \mathrm{mg})$ y tasa de crecimiento $(\mathrm{mm}$ $\left.\mathrm{d}^{-1}, \mathrm{mg} \mathrm{d}^{-1}\right)$.

\section{Bioensayo III. Reto experimental de juveniles de Argopec- ten ventricosus con Vibrio alginolyticus (supervivencia y actividad de la enzima superóxido dismutasa (SOD))}

Inmediatamente después de finalizar el bioensayo con juveniles, y 24 horas después de la última dosis de tratamiento, se tomaron 30 juveniles (72 días de edad; 5-6 $\mathrm{mm}$ altura de la concha; 20-27 mg peso húmedo) de cada uno de los ocho grupos experimentales, por duplicado, y se colocaron en frascos Erlenmeyer de $500 \mathrm{~mL}$ llenados con 300 $\mathrm{mL}$ de agua de mar filtrada $(1 \mu \mathrm{m})$ y esterilizada con UV. Los juveniles se infectaron con una única dosis de $V$. alginolyticus CAIM57 a $10 \times 10^{6}$ UFC $\mathrm{mL}^{-1}$. La dosis fue determinada mediante el cálculo de la dosis letal media (DL50) usando el método probit (Finney 1971). Adicionalmente a los ocho grupos se incluyó un control negativo (CTRL-; juveniles sin tratamiento y sin infectar), con la finalidad de evitar falsos positivos de la prueba por otros factores que puedan influir en la supervivencia de los juveniles en el reto. Durante el bioensayo los juveniles se alimentaron diariamente con la microalga I. galbana a $150 \times 10^{3}$ células $\mathrm{mL}^{-1}$. El bioensayo tuvo una duración de $96 \mathrm{~h}$.

Para este experimento se evaluó la supervivencia (\%) y la producción de la SOD antes de la infección y 48,72 y 96 $\mathrm{h}$ después del reto con $V$. alginolyticus, de cada uno de los tratamientos. Para determinar la SOD de cada tratamiento, se pesaron tejidos blandos $(100 \mathrm{mg})$ de seis juveniles y se agregaron $500 \mathrm{~mL}$ de tampón fosfato $(\mathrm{pH} 7,5)$. Los tejidos se homogeneizaron y se centrifugaron a $9327 \mathrm{~g}$ durante $10 \mathrm{~min}$ a $4^{\circ} \mathrm{C}$, recuperando el sobrenadante y almacenándolo a $20^{\circ} \mathrm{C}$ hasta un análisis adicional. La actividad de SOD se determinó con un kit comercial (SOD Assay Kit-WST \# 19160, SigmaAldrich). Los resultados se expresaron como porcentaje de inhibición del complejo WST-1 (water-soluble tetrazolium salt), un tinte cromogénico artificial formado por la reacción (Peskin y Winterbourn, 2000).

\section{Análisis estadístico}

Para cada grupo de datos se determinó la normalidad usando la prueba no paramétrica Kolmogorov-Smirnov y la homogeneidad de varianzas con la prueba de Levene. Se realizaron ANOVAS de un factor para identificar diferencias significativas entre los tratamientos, en el crecimiento y composición bioquímica de los juveniles, así como en la supervivencia y actividad de la SOD durante el reto experimental. Además, se realizó una análisis post hoc usando la prueba de Tukey (DHS) para conocer la diferencia entre los grupos. El nivel de significancia se fijó en $P<0.05$ para todos los análisis.

\section{RESULTADOS}

Bioensayo I. Supervivencia, crecimiento y fijación larvaria

En el día 7, se observaron diferencias significativas, la mayor supervivencia larvaria (Fig.1) se presentó en el tratamiento MIX-LBC2 (89\%) y AMP (91\%) comparado con MIX-LC1 (28\%). Los tratamientos, MIX-LBC2 y MIX-BC2 favorecieron la supervivencia ( 89 to $71 \%$ ).

En el crecimiento absoluto y tasa de crecimiento (Tabla 1) de larvas, se observaron diferencias significativas. El tratamiento con las cepa MIX-LBC2 presentó el mayor crecimiento de la concha $(147 \pm 3.7 \mu \mathrm{m})$ y mejor tasa de crecimiento (7.3 $\left.\pm 0.18 \mu \mathrm{m} \mathrm{d}^{-1}\right)$, comparadas con el grupo control CTRL (132 \pm $\left.3.2 \mu \mathrm{m} ; 6.4 \pm 0.20 \mu \mathrm{m} \mathrm{d}^{-1}\right)$. El menor crecimiento se observó en MIX-LC1 $\left(125 \pm 1.8 \mu \mathrm{m} ; 4.1 \pm 0.31 \mu \mathrm{m} \mathrm{d}^{-1}\right)$.

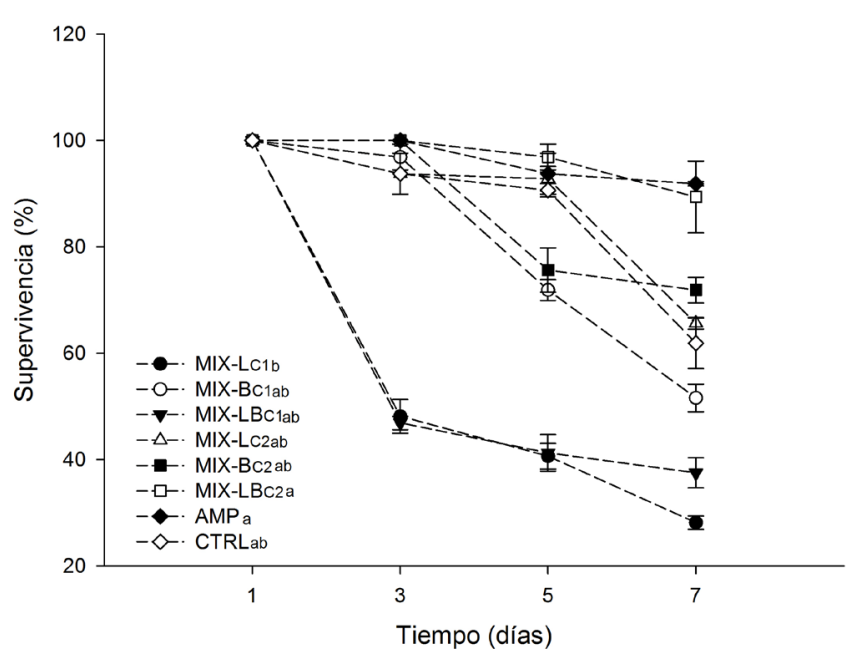

Figura 1. Porcentaje de supervivencia de la almeja Catarina (Argopecten ventricosus) tratadas con dos concentraciones, (C1): $1 \times 10^{5} \mathrm{UFC} \mathrm{mL}^{-1} \mathrm{y}$ (C2): $1 \times 10^{6}$ UFC mL ${ }^{-1}$, de las mezclas MIX-L (RL5+C3); MIX-B (PB1-1+PB15+PB1-6); MIX-LB (MIX-L+MIX-B), el antibiótico AMP (ampicilina a 10 ppm); y CTRL (sin tratamiento). Los datos representan la media $\pm D E$. Letras distintas denotan diferencias significativas entre tratamientos (Tukey DHS). Figure 1. Percentage of survival of the Catarina scallop (Argopecten ventricosus) treated with two concentrations, (C1): $1 \times 10^{5} \mathrm{CFU} \mathrm{mL}^{-1}$ and (C2): 1 $\times 10^{6} \mathrm{CFU} \mathrm{mL}^{-1}$, of the mix strains MIX-L (RL5 + C3); MIX-B (PB1-1 + PB1-5 + PB1-6); MIX-LB (MIX-L + MIX-B), the antibiotic AMP (ampicillin at $10 \mathrm{ppm}$ ); and CTRL (without treatment). Data represents the mean \pm SD. Different letters denote significant differences between treatments (Tukey HSD). 
Tabla 1. Altura de la concha, tasa de crecimiento (altura y peso) y fijación de la almeja Catarina (Argopecten ventricosus) tratadas con mezclas de cepas probióticas.

Table 1. Shell height, growth rate (height and weight), and fixation of the Catarina scallop (Argopecten ventricosus) treated with probiotic mix strains.

\begin{tabular}{|c|c|c|c|c|c|c|}
\hline \multirow[b]{2}{*}{ Tratamiento } & \multicolumn{2}{|c|}{ Larvas } & \multicolumn{4}{|c|}{ Juveniles } \\
\hline & $\begin{array}{l}\text { Altura de la concha } \\
\qquad(\mu \mathrm{m})\end{array}$ & $\begin{array}{c}\text { Tasa de crecimiento } \\
\left(\mu \mathrm{m} \mathrm{día}{ }^{-1}\right)\end{array}$ & $\begin{array}{c}\text { Fijación } \\
(\%)\end{array}$ & $\begin{array}{l}\text { Altura de la concha } \\
\qquad(\mathrm{mm})\end{array}$ & $\begin{array}{c}\text { Tasa de crecimiento } \\
(\mathrm{mm} \mathrm{día-1)}\end{array}$ & $\begin{array}{c}\text { Tasa de crecimiento } \\
\left(\mathrm{mg} \mathrm{día}^{-1}\right)\end{array}$ \\
\hline MIX-LC1 & $125 \pm 1.8^{C}$ & $4.1 \pm 0.31^{C}$ & 0.8 & $5.6 \pm 0.09^{a b}$ & $0.04 \pm .008$ & $0.33 \pm .007$ \\
\hline MIX-B C1 & $126 \pm 2.6^{c}$ & $4.6 \pm 0.47^{b c}$ & 0.8 & $5.2 \pm 0.07^{b c}$ & $0.02 \pm .002$ & $0.35 \pm .05$ \\
\hline MIX-LB C1 & $128 \pm 2.1^{C}$ & $4.7 \pm 0.13^{b c}$ & 0.5 & $5.4 \pm 0.08 \mathrm{abc}$ & $0.03 \pm .002$ & $0.58 \pm .02$ \\
\hline MIX-L C2 & $132 \pm 2.3^{b c}$ & $4.8 \pm 0.33^{b c}$ & 0.4 & $5.7 \pm 0.12^{a}$ & $0.04 \pm .001$ & $0.54 \pm .05$ \\
\hline MIX-B C2 & $136 \pm 2.8^{a b c}$ & $6.4 \pm 0.22^{a b c}$ & 0.7 & $5.6 \pm 0.07^{a}$ & $0.04 \pm .001$ & $0.46 \pm .01$ \\
\hline MIX-LB C2 & $147 \pm 3.7^{a}$ & $7.3 \pm 0.18^{a}$ & 10 & $5.4 \pm 0.10^{a b c}$ & $0.03 \pm .005$ & $0.31 \pm .02$ \\
\hline AMP & $144 \pm 3.3^{a b}$ & $6.2 \pm 0.25^{\mathrm{ab}}$ & 7.5 & $5.3 \pm 0.09 a b c$ & $0.03 \pm .03$ & $0.42 \pm .02$ \\
\hline CTRL & $132 \pm 3.2^{b c}$ & $6.4 \pm 0.20^{b c}$ & 2.2 & $5.0 \pm 0.10^{C}$ & $0.02 \pm .01$ & $0.52 \pm .01$ \\
\hline
\end{tabular}

MIX-L: RL5 + C3; MIX-B: PB1-1 + PB1-5 + PB1-6; MIX-LB: MIX-L + MIX-B; AMP: Ampicilina y CTRL: grupo control. Dos concentraciones, C1: $1 \times 10^{5}$ UFC mL ${ }^{-1}$ y C2: 1

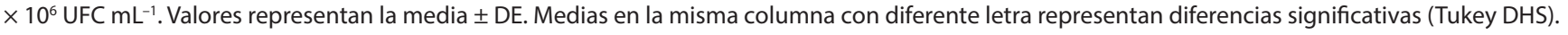
MIX-L: RL5 + C3; MIX-B: PB1-1 + PB1-5 + PB1-6; MIX-LB: MIX-L + MIX-B; AMP: Ampicillin and CTRL: control group. Two concentrations, C1:1 $\times 10^{5}$ CFU mL ${ }^{-1}$ and $\mathrm{C} 2: 1 \times 10^{6} \mathrm{CFU} \mathrm{mL} \mathrm{L}^{-1}$. Values represent the mean \pm SD. Means in the same column with different letters represent significant differences (Tukey HSD).

La fijación larvaria comenzó en el día 14 y presentó una duración de $5-6$ días. Al final, los mayores porcentajes de fijación se presentaron con el tratamiento MIX-LBC2 (10\%); y los porcentajes más bajos se observaron en el tratamiento MIX-LC2 (0.4\%) (Tabla 1).

Bioensayo II. Supervivencia, crecimiento y composición bioquímica de juveniles de Argopecten vetnricosus

La supervivencia fue del $100 \%$ en todos los tratamientos hasta el día 21 del experimento. Los juveniles presentaron mayor crecimiento de manera significativa (Tabla 1) cuando fueron tratados con MIX-LC2 $(5.7 \pm 0.12 \mathrm{~mm} ; 0.04 \pm .001 \mathrm{~mm}$ $\left.\mathrm{d}^{-1}\right)$, comparado con el grupo control $(5.03 \pm 0.10 \mathrm{~mm} ; 0.02$ $\mathrm{mm} \mathrm{d}^{-1}$ ). Así mismo, presentaron mayor peso (Fig. 2; Tabla 1) con los tratamientos MIX-LBC1 $(25,6 \pm 0.05 \mathrm{mg} ; 0.58 \mathrm{mg}$ día $\left.{ }^{-1}\right)$ y MIX-LC2 $\left(24.8 \pm 0.7 \mathrm{mg} ; 0.54 \mathrm{mg} \mathrm{día}^{-1}\right)$, comparado con el control $\left(24.3 \pm 0.1 \mathrm{mg} ; 0.52 \mathrm{mg} \mathrm{día}^{-1}\right)$. El tratamiento con menor peso fue MIX-LBC2 (20 $\left.\pm 0.2 \mathrm{mg} ; 0.31 \mathrm{mg} \mathrm{día}^{-1}\right)$.

\section{Bioensayo III. Supervivencia y actividad de la SOD en juveniles de Argopecten ventricosus retados con V. algi- nolyticus CAIM57}

Posterior a las $72 \mathrm{~h}$ de exposición a $V$. alginolyticus, la supervivencia de los juveniles fue del 100\% en CTRL (-), $0 \%$ en el CTRL, y entre $85-95 \%$ en todos los juveniles tratados con las mezclas de cepas probióticas (Fig.3). A las 96 h, la supervivencia fue significativamente mayor en los juveniles tratados con MIX-BC2 (85\%) y menor (5\%) in MIX-LC2.

Antes y después del reto experimental, la actividad de la enzima SOD presentó diferencias significativas, observando mayor porcentaje (inicial) en el tratamiento con MIX-BC1 (83\%) y MIX-BC2 (78 \%) y menor porcentaje con MIX-LC2 (44\%) y AMP (46\%). Comparado con el grupo control (15\%), la producción de SOD al finalizar las 48h de la infección, se incrementó en todos los tratamientos con las mezclas de cepas, particularmente con MIX-LC2 (58\%) y MIX-BC2 (56\%). A las $72 \mathrm{~h}$, la SOD se incrementó con el tratamiento MIX-BC2 (83\%) y MIX-BC1 (83\%) y a las $96 \mathrm{~h}$ en estos mismos tratamientos disminuyó la actividad de la enzima $60 \%$ y $56 \%$ respectivamente (Fig.4).

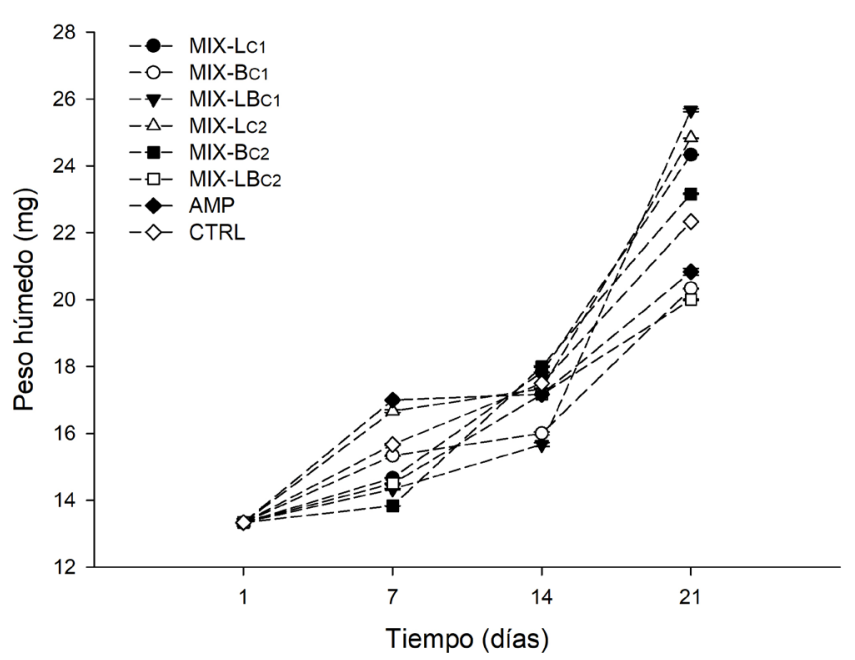

Figura 2.- Peso húmedo de la concha de juveniles de la almeja Catarina tratados con las mezclas de las cepas probióticas seleccionadas. Los puntos y las líneas verticales representan la media $\pm D E$. Letras distintas denotan diferencias significativas entre tratamientos (Tukey DHS).

Figure 2.- Juveniles shell wet weight of Catarina scallop treated with selected probiotic mix strains. Points and vertical lines represent the mean \pm SD. Different letters denote significant differences between treatments (Tukey HSD). 


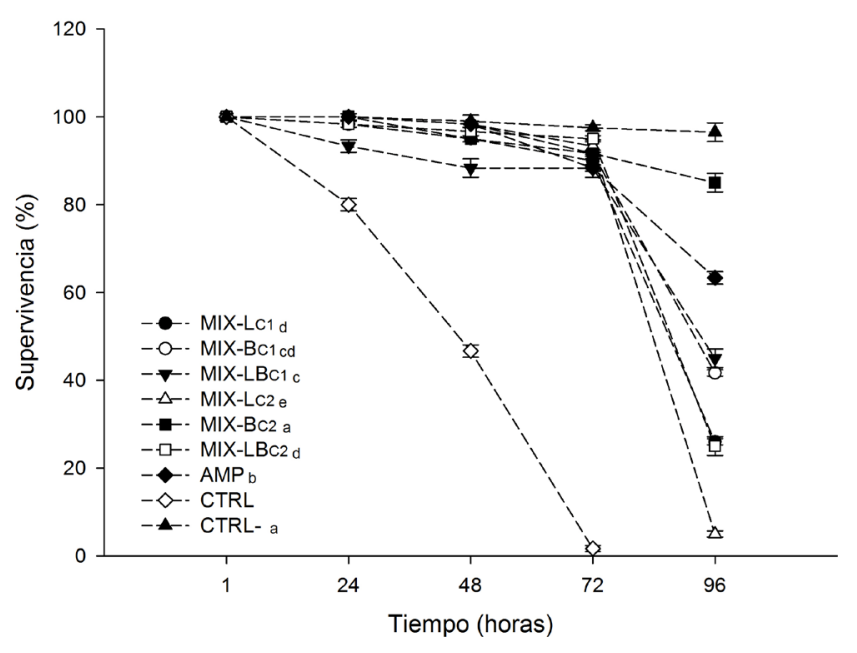

Figura 3.- Supervivencia de juveniles de la almeja Catarina tratados con las mezclas de cepas probióticas seleccionadas y retadas con Vibrio alginolyticus. CTRL(-) representa a juveniles sin tratamiento y sin retar y CTRL (+) representa a juveniles sin tratamiento y retados. Los puntos y las líneas verticales representan la media $\pm \mathrm{DE}$. Letras distintas denotan diferencias significativas entre tratamientos (Tukey DHS).

Figure 3.- Survival of Catarina scallop juveniles treated with selected probiotic mix strains and challenged with Vibrio alginolyticus. CTRL (-) represents juveniles without treatment and without challenge and CTRL (+) represents juveniles without treatment and challenged. Points and the vertical lines represent the mean \pm SD. Different letters denote significant differences between treatments (Tukey HSD).

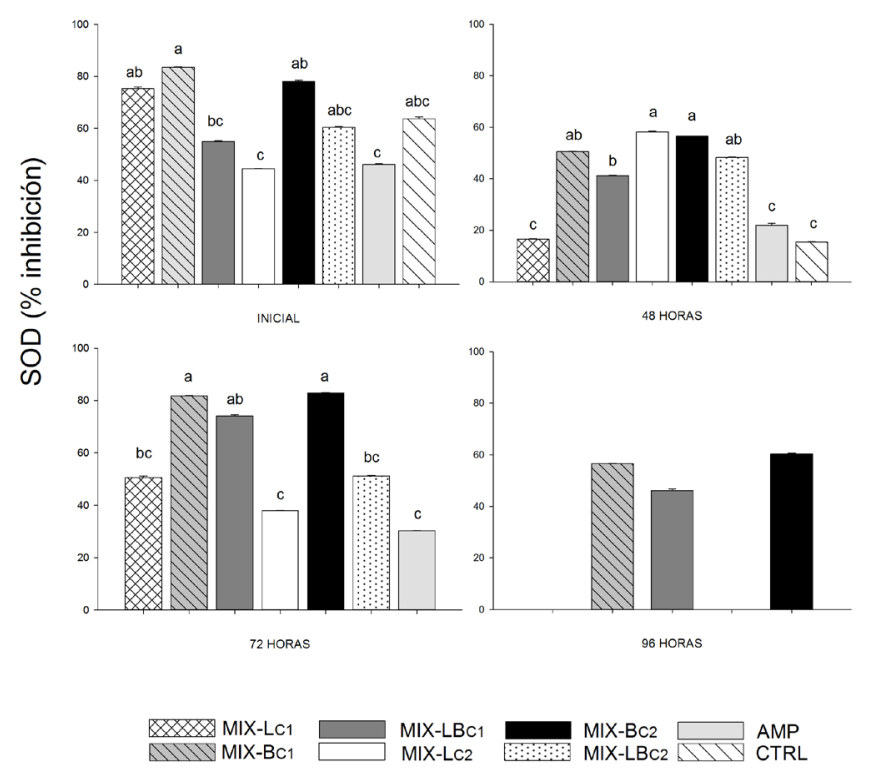

Figura 4.- Actividad de la SOD determinada por el porcentaje de inhibición de la formación del complejo WST-1 (water-soluble tetrazolium) Formazan en juveniles de almeja Catarina tratados previamente con la mezcla de bacterias probióticas seleccionadas y posteriormente retados durante 96 horas con V. alginolyticus (cepa CAIM57).

Figure 4.- SOD activity determined by the percentage of inhibition of the WST-1 complex (water-soluble tetrazolium) Formazan formation in Catarina scallop juveniles previously treated with the selected probiotic mix strains, and subsequently challenged for 96 hours with V. alginolyticus (strain CAIM57).

\section{DISCUSIÓN}

En el presente trabajo la mayoría de los tratamientos con mezcla de cepas probióticas en diferentes concentraciones mejoraron el estado general de $A$. ventricosus, produciendo un efecto de manera diferente en cada etapa de desarroIlo. En larvas la mezcla con Bacillus spp. y Lactobacillus spp. (MIX-LBC2) mejoraron significativamente la supervivencia y el crecimiento en talla y peso, comparada con la mezcla de solo Lactobacillus spp. (MIX-LC1) y Bacillus spp. (MIX-BC1). AI respecto podemos decir que las cepas probióticas mixtas son más eficientes que los probióticos en base a una sola cepa (Verschuere et al., 2000; Hai et al. 2009). Este efecto puede ser causado por un efecto sinérgico entre las cepas y entre los metabolitos secundarios producidos por las cepas. Este sinergismo incluye la mejora en absorción de nutrientes que resulta favorable para incrementar el crecimiento del hospedero (Irianto y Austin, 2002). Así mismo, esta mezcla ha demostrado resultados benéficos. Por ejemplo, la mezcla de las cepas probióticas Bacillus subtilis y Lacobacillus acidophilus incrementan la supervivencia y mejoran el crecimiento de la tilapia del Nilo oreochromis niloticus (Aly et al., 2008) así mismo, se encontró un alto crecimiento, una mejor tasa de eficiencia proteica, digestibilidad y retención de nutrientes en alevines de Labeo rohita alimentados con una mezcla probiótica de B. subtilis, L. lactis y Saccharomyces cerevisiae (Mohapatra et al., 2012). En el presente trabajo la fijación larvaria de $A$. ventricosus se vio favorecida por el uso de esta mezcla de especies bacterianas, al respecto se ha documentado que existe una relación directa de las bacterias en estos procesos que favorecen la adhesión. Esto se evidenció en los primeros trabajos realizados por Weiner y Colwell (1982), demostrando que una bacteria marina pigmentada (Shewanella colwelliana) favorece la fijación de larvas de $C$. virginica y C. gigas, por medio de mecanismos como la formación de biofilms y producción de exopolisacáridos. Así mismo se han observado otros muchos efectos benéficos utilizando mezclas de bacterias como probióticos, por ejemplo, una cepa de Vibrio sp. favoreció la ingestión de microalgas en larvas de $A$. purpuratus (Avendaño y Riquelme, 1999) y la mezcla de esta cepa con una Pseudomona sp. y un Bacillus sp., favorecieron el desarrollo larvario sin el uso de antibióticos (Riquelme et al., 2001).

Para el caso de juveniles el mejor tratamiento sucedió con la mezcla de Lactobacillus spp., caso contrario a lo visto en larvas. Al respecto en juveniles de C. sikamea tratados con la mezcla de Lactobacillus, se muestra un incremento en la concentración de proteínas (Abasolo-Pacheco et al., 2016), lo cual puede indicar una mejor asimilación de los nutrientes, favoreciendo el crecimiento en biomasa. En el presente trabajo se encontró el mismo efecto, favoreciendo el crecimiento de juveniles de $A$. ventricosus, sugiriendo una mejor asimilación y digestión de nutrientes tratados con las mezclas de Lactobacillus, esto podría explicarse por el efecto de los metabolitos secundarios producidos por este género (Balcazar et al., 2006; Nevejan et al., 2018). Por ejemplo, en larvas de la dorada (gilthead sea bream) Sparus aurata, se 
mejoró el crecimiento y supervivencia, así como la actividad de las enzimas digestivas, al ser tratadas con una mezcla de Lactobacillus probióticos (Suzer et al., 2008).

En referencia al uso de las concentraciones de mezclas probióticas usadas en este trabajo se observó que el uso de concentraciones de $1 \times 10^{6} \mathrm{CFU} \mathrm{mL} \mathrm{mL}^{-1}$ fue la más efectiva, en este sentido Vine et al. (2006), señalan que concentraciones de probióticos entre $1 \times 10^{4}$ células $\mathrm{mL}^{-1}$ y $1 \times 10^{6}$ células $\mathrm{mL}^{-1}$ son suficientes en el agua de cultivo para lograr que las bacterias probióticas se establezcan como parte de la microbiota intestinal del hospedero. En nuestro estudio, todos los tratamientos mostraron mejor rendimiento en cuanto a supervivencia y crecimiento en larvas de $A$. ventricosus, al elevar las concentraciones de las mezclas de $1 \times 10^{5} \mathrm{UFC} \mathrm{mL}^{-1}$ a $1 \times 10^{6}$ UFC $\mathrm{mL}^{-1}$, particularmente MIX-BC2. Resultados similares se han observado en larvas de $C$. corteziensis en donde la supervivencia mejoró al subir las concentraciones de una mezcla de cepas probióticas (Pseudomonas aeruginosa YC58 y Burkholderia cepacia Y021) de $1 \times 10^{4}$ UFC $\mathrm{mL}^{-1}$ a 1 $\times 10^{5} \mathrm{UFC} \mathrm{mL}^{-1}$ (Campa-Córdova et al., 2011). Es importante mencionar que esta misma mezcla de microorganismos pero en concentraciones de $1 \times 10^{9} \mathrm{UFC} \mathrm{mL} \mathrm{L}^{-1}$ no mostraron efectos benéficos (Granados-Amores et al., 2012) confirmando que el uso de la concentraciones afecta directamente el efecto probiótico de las mezclas de microorganismos.

Finalmente, se observó que durante el reto experimental con V. alginolyticus CAIM57, la mezcla de Bacillus spp. (MIX-BC2) mostró mejores resultados de supervivencia en juveniles de $A$. ventricosus. Este efecto se ha observado contra otros patógenos usando mezclas de Bacillus, como la mezcla de $B$. subtilis y $B$. licheniformis controlan a los patógenos Yersinia ruckeri y Lactococcus garvieae (Raida et al., 2003), y la mezcla de dos cepas de B. subtilis al patógeno $V$. harveyi (Zokaeifar et al., 2012). A pesar de que las tres cepas no presentaron actividad antagónica in vitro contra $V$. alginolyticus (Abasolo-Pacheco et al., 2016), si presentaron mejor protección durante el reto con los juveniles en este estudio. Los probióticos multiespecíficos y de múltiples cepas refuerzan el mecanismo de defensa contra diversas enfermedades infecciosas (Kesarcodi-Watson et al., 2012). Resultados similares se encontraron al realizar un reto experimental con $V$. tubiashii en juveniles de C. virginica (Karim et al., 2013), en donde la cepa probiótica utilizada no presenta actividad antagónica in vitro pero si transfiere amplia protección a larvas y juveniles tratados, sugiriendo que la protección transferida por la cepa probiótica no es resultado de la actividad antibiótica de la cepa sino por estimulación del sistema inmune del organismo (Verschuere et al., 2000). Este mismo efecto se puede sugerir en nuestro estudio y se ve apoyado por la actividad de la SOD en los tratamientos evaluados y su relación con la supervivencia durante el reto, sobre todo en MIX-BC2, confirmando que las cepas del género Bacillus son efectivas para mejorar la respuesta del sistema immune en organismos marinos (Rengpipat et al., 2000; Eissa et al., 2018). Al respecto, parece claro que cuando las bacterias patógenas entran al cuerpo de los bivalvos se desencadenan una serie de res- puesta del sistema inmune, como producción de enzimas hidrolíticas, factores antimicrobianos y especies reactivas de oxígeno (EROs), con la finalidad de neutralizar y destruir a los agentes invasores (Vargas-Albores y Barracco, 2001). La SOD ha sido utilizada como indicadora de respuesta inmune en invertebrados marinos (Luna-Gónzalez et al., 2003; CampaCórdova et al., 2009), esta enzima juega un papel crucial en la defensa de bivalvos (Song et al., 2010), llevando al incremento o disminución de su actividad (Matsuda et al., 2003). Finalmente es importante mencionar que la importancia de la SOD en la respuesta inmune, así como el papel que desempeña en la protección de las células en diferentes retos se ha reportado en diferentes trabajos (Granados-Amores et al., 2012; Genard et al., 2014; Le Bris et al., 2015).

\section{CONCLUSIONES}

Los resultados obtenidos sugieren que la eficiencia y modo de acción de la mezcla de probióticos puede variar entre cepas de una misma especie. En la supervivencia, crecimiento y fijación de larvas el mejor fue el tratamiento MIX$\mathrm{LBC2}$; respecto al crecimiento en talla y peso de juveniles, la mezcla de Lactobacillus (MIX-LC2) fue la más eficiente. Las cepas de Bacillus spp. (en mezcla y de forma individual) no presentaron actividad antagónica in vitro contra los Vibrios evaluados, a excepción del tratamiento MIX-B que confirió la mayor protección durante el reto y generó los mayores niveles de SOD, sugiriendo la estimulación del sistema inmune.

\section{AGRADECIMIENTOS}

Al personal del CIBNOR: Norma Ochoa-Alvarez y Yenni Morales-Cristobal por el apoyo en el cultivo de microorganismos; Delfino Barajas-Frías y Pablo Ormart-Castro por el apoyo en el cultivo de larvas y juveniles; Julian Garzón-Favela en el cultivo de microalgas; Adan Altamirano (Sistemar) por la donación de reproductores de la almeja Catarina. A los proyectos del CIBNOR, SEP-CONACYT (243532) y PROINNOVA-CONACYT (199788).

\section{REFERENCIAS}

Abasolo-Pacheco, F., Saucedo, P. E., Mazón-Suástegui, J. M., Tovar-Ramírez, D., Araya, R., Ramírez-Orozco, J. M., \& Campa-Córdova, Á. I. (2016). Isolation and use of beneficial microbiota from the digestive tract of lions-paw scallop Nodipecten subnodosus and winged pearl oyster Pteria sterna in oyster aquaculture. Aquaculture research. $47(10)$ : 3042-3051.

Alós, J. I. (2015). Resistencia bacteriana a los antibióticos: una crisis global. Enfermedades infecciosas y microbiología clínica, 33(10), 692-699.

Aly, S. M., Ahmed, Y. A. G., Ghareeb, A. A. A. y Mohamed, M. F. 2008. Studies on Bacillus subtilis and Lactobacillus acidophilus, as potential probiotics, on the immune response and resistance of Tilapia nilotica (Oreochromis niloticus) to challenge infections. Fish \& shellfish immunology. 25(1-2): 128-136. 
Avendaño, R. E., \& Riquelme, C. E. (1999). Establishment of mixed-culture probiotics and microalgae as food for bivalve larvae. Aquaculture Research, 30(11-12), 893-900.

Granados-Amores, A., Campa-Córdova, Á. I., Araya, R., MazónSuástegui, J. M., \& Saucedo, P. E. (2012). Growth, survival and enzyme activity of lions-paw scallop (Nodipecten subnodosus) spat treated with probiotics at the hatchery. Aquaculture Research, 43(9), 1335-1343.

Balcázar, J. L., Vendrell, D., de Blas, I., Ruiz-Zarzuela, I., Muzquiz, J. L.y Girones, O. 2008. Characterization of probiotic properties of lactic acid bacteria isolated from intestinal microbiota of fish. Aquaculture. 278(1-4): 188-191.

Cáceres-Martínez, J., \& Vásquez-Yeomans, R. (2008). La patología en moluscos bivalvos: principales problemas y desafíos para la producción de bivalvos en América Latina. FAO Actas de Pesca y Acuicultura.

Campa-Córdova, A. I., Núñez-Vázquez, E. J., Luna-González, A., Romero-Geraldo, M. J. y Ascencio, F. 2009. Superoxide dismutase activity in juvenile Litopenaeus vannamei and Nodipecten subnodosus exposed to the toxic dinoflagellate Prorocentrum lima. Comparative Biochemistry and Physiology Part C: Toxicology \& Pharmacology. 149(3): 317322.

Cordero, H., Esteban, M. Á. y Cuesta, A. 2014. Use of probiotic bacteria against bacterial and viral infections in shellfish and fish aquaculture. In Sustainable Aquaculture Techniques. InTech.

Eissa, N., Wang, H. P., Yao, H., \& Abou-ElGheit, E. (2018). Mixed Bacillus species enhance the innate immune response and stress tolerance in yellow perch subjected to hypoxia and air-exposure stress. Scientific reports, 8(1), 6891.

Finney, D. J. 1971. Probit Analysis: 3d Ed. Cambridge University Press.

Gatesoupe, F. J. 2002. Probiotic and formaldehyde treatments of Artemia nauplii as food for larval pollack, Pollachius pollachius. Aquaculture. 212(1-4): 347-360.

Genard, B., O. Larouche, J.L. Nicolas, P. Miner, M.L. Beaudin, R. Tremblay. 2014. Effect of the probiotic strain Phaeobacter gallaeciensis after bacterial challenge on the complete larval development of Pecten maximus. Aquat Living Resour. 27(01):27-34.

Granados-Amores, A., A.I. Campa-Córdova, R. Araya, J.M. MazónSuástegui, P.E. Saucedo. 2012. Growth, survival and enzyme activity of lions-paw scallop (Nodipecten subnodosus) spat treated with probiotics at the hatchery. Aquac Res. 43(9):1335-1343.

Irianto, A. y Austin, B. 2002. Probiotics in aquaculture. Journal of fish diseases. 25(11): 633-642.

Karim, M., Zhao, W., Rowley, D., Nelson, D. y Gomez-Chiarri, M. 2013. Probiotic strains for shellfish aquaculture: protection of eastern oyster, Crassostrea virginica, larvae and juveniles againsl bacterial challenge. Journal of Shellfish Research. 32(2): 401-408.

Kesarcodi-Watson, A., Kaspar, H., Lategan, M. J. y Gibson, L. 2008. Probiotics in aquaculture: the need, principles and mechanisms of action and screening processes. Aquaculture. 274(1): 1-14.

Kesarcodi-Watson, A., Miner, P., Nicolas, J. L. y Robert, R. 2012. Protective effect of four potential probiotics against pathogen-challenge of the larvae of three bivalves: Pacific oyster (Crassostrea gigas), flat oyster (Ostrea edulis) and scallop (Pecten maximus). Aquaculture. 344: 29-34.
Lawrence, R. y Jeyakumar, E. 2013. Antimicrobial resistance: A cause for global concern. BMC proceedings. 7(3): S1.

Le Bris, C., G. Richard, C. Paillard, C. Lambert, C. Seguineau, O. Gauthier, F. Pernet, F. Guérard. 2015. Immune responses of phenoloxidase and superoxide dismutase in the manila clam Venerupis philippinarum challenged with Vibrio tapetisPart I: Spatio-temporal evolution of enzymes' activities postinfection. Fish Shellfish Immun. 42(1):16-24.

Luna-González, A., Maeda-Martínez, A. y Ascencio-Valle, F. 2002. Comparative susceptibility of veliger larvae of tour bivalve mollusks to a Vibrio alginolyticus strain. Diseases of Aquatic Organisms. 49(3): 221-226.

Luna-González, A., Maeda-Martı冈nez, A. N., Vargas-Albores, F., Ascencio-Valle, F. y Robles-Mungaray, M. 2003. Phenoloxidase activity in larval and juvenile homogenates and adult plasma and haemocytes of bivalve molluscs. Fish \& shellfish immunology. 15(4): 275-282.

Maeda-Martínez, A.N. 2008. Estado actual del cultivo de bivalvos en México. En: Lovatelli, A. et al. (Ed.) Estado actual del cultivo y manejo de moluscos bivalvos y su proyección futura. factores que afectan su sustentabilidad en América Latina. Taller Técnico Regional de la FAO, 20-24 de agosto de 2007, Puerto Montt, chile. FAO Actas de Pesca y Acuicultura. 12: 91-100.

Mazón-Suástegui, J.M. 2005. Biología y cultivo de la almeja Catarina Argopecten ventricosus (Sowerby II, 1842). Ph. D. dissertation. University of Barcelona, Barcelona, Spain.

Mohapatra, S., Chakraborty, T., Prusty, A. K., Das, P., Paniprasad, K. y Mohanta, K. N. 2012. Use of different microbial probiotics in the diet of rohu, Labeo rohita fingerlings: effects on growth, nutrient digestibility and retention, digestive enzyme activities and intestinal microflora. Aquaculture Nutrition. 18(1): 1-11.

Nevejan, N., De Schryver, P., Wille, M., Dierckens, K., Baruah, K., \& Van Stappen, G. (2018). Bacteria as food in aquaculture: do they make a difference?. Reviews in Aquaculture, 10(1), 180-212.

Peskin, A. V. y Winterbourn, C. C. 2000. A microtiter plate assay for superoxide dismutase using a water-soluble tetrazolium salt (WST-1). Clinica chimica acta. 293(1-2): 157-166.

Raida, M. K., Larsen, J. L., Nielsen, M. E. y Buchmann, K. 2003. Enhanced resistance of rainbow trout, Oncorhynchus mykiss (Walbaum), against Yersinia ruckeri challenge following oral administration of Bacillus subtilis and B. licheniformis (BioPlus2B). Journal of Fish Diseases. 26(8): 495-498.

Rengpipat, S., Rukpratanporn, S., Piyatiratitivorakul, S. y Menasaveta, P. 2000. Immunity enhancement in black tiger shrimp (Penaeus monodon) by a probiont bacterium (Bacillus S11). Aquaculture. 191(4): 271-288.

Romalde, J. L. (2016). Héroes y villanos: bacterias asociadas al cultivo de moluscos. Revista AquaTIC, (37).

Sánchez-Ortiz, A. C., Luna-González, A., Campa-Córdova, Á. I., Escamilla-Montes, R., Flores-Miranda, M. D. C. y MazónSuástegu, J. M. 2015. Isolation and characterization of potential probiotic bacteria from pustulose ark (Anadara tuberculosa) suitable for shrimp farming. Latin American Journal of Aquatic Research. 43(1): 123-136.

Suzer, C., Çoban, D., Kamaci, H. O., Saka, Ş., Firat, K., Otgucuoğlu, Ö. y Küçüksari, H. 2008. Lactobacillus spp. bacteria as probiotics in gilthead sea bream (Sparus aurata, L.) larvae: effects on growth performance and digestive enzyme activities. Aquaculture. 280(1-4): 140-145. 
Vargas-Albores, F., M.A. Barracco. 2001. Mecanismos de defensa de los moluscos bivalvos con énfasis en pectinídeos. En: Maeda-Martínez, A.N. (ed.) Los Moluscos Pectínidos de Iberoamérica. Ciencia y Acuicultura. Limusa, México. pp. 127-140.

Verschuere, L., Rombaut, G., Sorgeloos, P. y Verstraete, W. 2000. Probiotic bacteria as biological control agents in aquaculture. Microbiology and molecular biology reviews. 64(4): 655-671.

Vine, N.G., Leukes, W.D. y Kaiser, H. 2006. Probiotics in marine larviculture. FEMS microbiology reviews. 30(3): 404-427.

Weiner, R.M., y Colwell, R.R. 1982. Induction of settlement and metamorphosis in Crassostrea virginica by a melaninsynthesizing bacterium. Sea Grant Program, University of Maryland.
Yeh, S.P., Chiu, C.H., Shiu, Y.L., Huang, Z.L., \&Liu, C.H. (2014). Effects of diets supplemented with either individual or combined probiotics, Bacillus subtilis E20 and Lactobacillus plantarum 7-40, on the immune response and disease resistance of the mud crab, Scylla paramamosain (Estampador). Aquaculture research, 45(7), 1164-1175.

Zokaeifar, H., Balcázar, J. L., Kamarudin, M. S., Sijam, K., Arshad, A., y Saad, C. R. 2012. Selection and identification of nonpathogenic bacteria isolated from fermented pickles with antagonistic properties against two shrimp pathogens. The Journal of antibiotics. 65(6): 28. 